\title{
Resistant starch production in wheat bread: effect of ingredients, baking conditions and storage
}

\author{
Olga Amaral ${ }^{1,2} \cdot$ Catarina S. Guerreiro $^{2}$ Ana Gomes $^{3} \cdot$ Marília Cravo $^{2}$
}

Received: 14 January 2016 / Revised: 10 February 2016 / Accepted: 5 March 2016 / Published online: 22 March 2016

(C) Springer-Verlag Berlin Heidelberg 2016

\begin{abstract}
Resistant starch (RS) is defined as the sum of starch and products of starch degradation not absorbed in the small intestine of healthy individuals. RS appears to confer considerable health benefits. Processing conditions and ingredients may influence the formation of RS in foodstuffs. The aim of the present study was to investigate some of the factors that might influence RS formation in wheat bread, namely (1) formulation; (2) loaf size; (3) baking conditions; and (4) storage conditions. Seven bread formulations were prepared: reference recipe (control) and six experimental formulations. The effect of the loaf size and the baking conditions was also tested. Finally, the effect of storage was tested by keeping control breads under different storage conditions (temperature/time). Moisture and resistant starch contents were evaluated in all breads. A higher level of moisture in the dough and a larger loaf size enhanced the RS content. An extended baking process also favored significantly the formation of RS. Storing the bread at room temperature for 3 days was shown to further increase the RS content. It is possible to increase the RS content of bread by modifying the ingredients ratio and processing conditions.
\end{abstract}

Olga Amaral

olga.amaral@ipbeja.pt

1 Departamento de Tecnologias e Ciências Aplicadas, Instituto Politécnico de Beja, Campus do IPBeja, Apartado 6155, 7800-295 Beja, Portugal

2 Faculdade de Medicina, Universidade de Lisboa, Avenida Professor Egas Moniz, 1649-028 Lisbon, Portugal

$3 \mathrm{CBQF}$ - Centro de Biotecnologia e Química Fina Laboratório Associado, Escola Superior de Biotecnologia, Universidade Católica Portuguesa/Porto, Rua Arquiteto Lobão Vital, Apartado 2511, 4202-401 Porto, Portugal
Keywords Resistant starch · Wheat bread · Retrogradation $\cdot$ Storage

\section{Introduction}

Starch is present in plant foods and occurs as insoluble granules. By heating starch in the presence of water (50$70{ }^{\circ} \mathrm{C}$ ), irreversible changes occur-starch gelatinization [1]. The digestion of starch begins in the mouth by salivary amylase and is completed in the small intestine, resulting in glucose molecules that are absorbed directly from the intestinal mucosa. Before the early 1980s, starch was assumed to be fully hydrolyzed and absorbed in the small intestine. Studies undertaken to develop an assay method for the in vitro measurement of non-starch polysaccharides revealed that a portion of the ingested starch is not hydrolyzed and resists digestion. This fraction is called resistant starch (RS) [2].

Resistant starch has been defined as "the sum of starch and products of starch degradation not absorbed in the small intestine of healthy individuals" [3]. Classification of RS is generally made over five categories (RS1-RS5) based on the mechanism that contributes to their resistance to digestion $[4,5]$. RS1 is starch that escapes digestion because it is physically inaccessible by entrapment in a non-digestible matrix. The preparation and cooking process of food is of great importance for the RS1 content because these procedures can contribute to a disruption of cell walls $[4,6]$. RS2 consists of raw starch granules (ungelatinized) which have retained their crystal structure; therefore, they are not attacked by digestive enzymes. Most cooking procedures are able to gelatinize raw starches allowing the disappearance of RS2 in the food $[4,6]$. RS3 is present in most of starchy foods, which have been cooked and then 
cooled and stored for several hours up to several months. Upon cooling, starch polymer chains begin to reassociate as double helices and can form tightly packed structures stabilized by hydrogen bonding (retrogradation). The retrograded starch composed mainly of amylase is very stable. Amylopectin can also retrograde, although a much longer time is needed [4, 6, 7]. Because of its thermal stability, RS3 may be used as an ingredient in a wide variety of foods [6]. RS4 encompasses starches obtained by chemical treatments. It includes ethers and esters as well as cross-bonded starches that are resistant to small intestinal digestion [6]. RS5 is an inclusion complex formed by alpha-amylase with polar lipids [5]. These types of starch require higher temperatures for gelatinization and are more susceptible to retrogradation [8]. RS1, RS2 and RS3 can coexist in the same food. Beans, for example, contain both RS1 and RS3, whereas RS1 and RS2 are present in bananas. The natural types of RS are frequently destroyed when processed. Food processing which involves heat and moisture, in most cases, destroys RS1 and RS2, but may form RS3 [9].

Interest in RS research has increased during the last three decades. RS appears to confer considerable health benefits related to inflammatory bowel disease and bowel cancer, reduction in postprandial glycemia/insulinemia, improvement in insulin sensitivity, fat oxidation and satiety, and plays a prebiotic role [5, 7, 9-12]. RS is fermented by human colonic bacteria in the large intestine, resulting in the production of gases (carbon dioxide, methane and hydrogen) and short-chain fatty acids (SCFA). SCFA produced include butyrate, acetate and propionate, and it is thought that these fatty acids are mainly responsible for the beneficial effects of RS. There is also increasing interest in using RS to lower the energy value and available carbohydrate content while enhancing the fiber content of foods [10].

The amount of RS in food is low and is determined by the starch botanical source, extent of processing and storage conditions. The current interest lies in the fact that it is technically possible to increase the RS amounts in food. The content of RS formed during the processing of starchy foods is controlled by a number of factors such as water content, $\mathrm{pH}$, heating temperature and time, number of heating and cooling cycles, freezing and drying [13, 14]. Products based on starch with elevated amylose content revealed higher levels of RS, mainly retrograded amylose (RS3) [15]. Another possibility included the development of food products supplemented with RS as an ingredient, with no negative effects on the taste or texture of the final product. These food products have better consumer acceptability and greater palatability than those that are fortified with traditional dietary fibers such as whole wheat bread (dark color, reduced loaf volume and poor mouthfeel, that are negative attributes often associated with high-fiber breads) [7].
Bread, one of the most important dietary carbohydrate sources, generally contains small amounts of RS [15]. Bread products vary widely around the world, but the basic ingredients are cereal flour, water, yeast or another leavening agent and salt. The breadmaking process consists of three major stages: mixing, fermentation and baking. Processing conditions and ingredients may influence the formation of RS in bread. A low-temperature, long-timebaked product contained significantly higher amounts of RS than bread baked under ordinary conditions [16-18]. Higher water content in the dough appeared to stem in higher amounts of RS [15, 19]. Replacing part of the flour with flour from novel genotypes with high amylose content resulted in a higher content of RS in the baked breads [20]. The digestibility of starch is also reduced in the presence of lactic acid, either added or produced during sourdough fermentation [16, 20-22]. Although some studies demonstrated that storage of wheat bread did not affect the amount of RS [15, 19, 23], others were able to show that the content of RS increased during storage. In particular, in corn bread, cold storage seemed to support an increase in RS content [13, 18, 24].

The aim of the present study was to investigate some of the factors that might influence RS formation in traditional wheat bread, namely (1) formulation type; (2) loaf size; (3) baking conditions; and (4) storage temperature/time.

Several previous research works carried out in this area have not used the RS assay method currently approved by $\mathrm{AOAC}^{1}$ and by the AACC, ${ }^{2}$ used in this study. This work contributed to clarifying the effect of the studied variables and compares more recent studies that used this RS assay method.

\section{Materials and methods}

\section{Materials}

The following raw materials were used: commercial wheat flour (own brand Continente type 65, Portugal), commercial whole spelt flour (Próvida, Portugal), commercial compressed baker yeast (Mauri, Italy) and common table salt. These products were purchased from the Portuguese market.

All chemicals and reagents were of analytical grade and were obtained from Riedel-de-Haen, Panreac or Fisher Chemicals. The Megazyme Kit (Megazyme International Ireland Ltd) was acquired from Enzymatic (Lisbon, Portugal).

\footnotetext{
${ }^{1}$ Association of Official Analytical Chemists.

${ }^{2}$ American Association of Cereal Chemists.
} 
Table 1 Standard recipe and different formulation types tested in terms of flour-to-water ratio (LW and $\mathrm{HW}$ ), wheat flour replacement with spelt flour (S25 and S50), lactic acid addition (LA) and sourdough addition (SD)

\begin{tabular}{lrrrrrrr}
\hline & REF & LW & HW & S25 & S50 & LA & SD \\
\hline Common white wheat flour (g) & 1000 & 1000 & 1000 & 750 & 500 & 1000 & 1000 \\
Spelt wheat flour (g) & 0 & 0 & 0 & 250 & 500 & 0 & 0 \\
Water (ml) & 625 & 500 & 714 & 625 & 625 & 625 & 625 \\
Lactic acid (90 wt\%) (ml) & 0 & 0 & 0 & 0 & 0 & 16 & 0 \\
Sourdough (g) & 0 & 0 & 0 & 0 & 0 & 0 & 100 \\
Salt (g) & 15 & 15 & 15 & 15 & 15 & 15 & 15 \\
Baker's yeast (g) & 20 & 20 & 20 & 20 & 20 & 20 & 10 \\
\hline
\end{tabular}

$R E F$ traditional recipe, $L W 2.0: 1$ flour-to-water ratio, $H W$ 1.4:1 flour-to-water ratio, $S 2525 \%$ of spelt flour, $S 5050 \%$ of spelt flour, $L A$ addition of lactic acid, $S D$ addition of sourdough
Table 2 Standard recipe parameters and different conditions tested in terms of loaf size (W35, W140 and W400) and baking conditions (T150 and T120)

\begin{tabular}{lllllll}
\hline & REF & W35 & W140 & W400 & T150 & T120 \\
\hline $\begin{array}{l}\text { Weight (g) } \\
\text { Baking } \\
\text { conditions }\end{array}$ & 70 & 35 & 140 & 400 & 70 & 70 \\
\hline
\end{tabular}

\section{Production of bread formulations}

Seven different bread formulations were prepared based on the standard recipe and sequentially changing the parameters under study, as shown in Table 1.

All breads were prepared according to the traditional breadmaking procedure. For making the dough, the flour was mixed with warm water, yeast and salt in a dough mixer at the lowest speed for 5 min and, thereafter, mixed at medium speed for an additional $10 \mathrm{~min}$. The dough was allowed to ferment in a humidity chamber at $30{ }^{\circ} \mathrm{C}$ and relative humidity of $85 \%$ for $40 \mathrm{~min}$. The dough was manually molded into $84 \mathrm{~g}$ balls (70 g after baking) and proofed, in the same conditions, for another $40 \mathrm{~min}$. The breads were baked at $200{ }^{\circ} \mathrm{C}$ for $30 \mathrm{~min}$. After baking, the breads were cooled for $2 \mathrm{~h}$ at room temperature $\left(\approx 22{ }^{\circ} \mathrm{C}\right)$ and were further analyzed for RS and moisture within the same day.

A second experiment involved testing the effect of loaf size and baking conditions on the final resistant starch content of bread. Different loaf sizes and baking conditions were used to prepare a set of 6 breads using as basis the standard recipe, as shown in Table 2.

In order to assess the influence of storage conditions on the formation of RS, breads were prepared according to the REF formulation. After cooling, breads were kept in scaled bags under different storage conditions: room temperature $\left(22^{\circ} \mathrm{C}\right), 4$ and $-20{ }^{\circ} \mathrm{C}$ for $1,3,5$ and 7 days.

Each batch treatment was replicated four times. Each sample of each batch, consisting of four bread loaves (except W140 - 2 bread loaves—and W400 - 1 bread loaf), was analyzed in duplicate.

\section{Determination of moisture and resistant starch}

\section{Moisture}

Moisture content of the bread was determined according to the official method AACC 44-15.02/AOAC 925.10. This method determines moisture content as loss in weight of a sample when heated under specified conditions. In a first stage, the bread sample (an aliquot of approximately $140 \mathrm{~g}$ ) is heated at $65{ }^{\circ} \mathrm{C}$ for $16 \mathrm{~h}$, and at a second stage, an amount of $5-7 \mathrm{~g}$ is heated in an air oven at $103{ }^{\circ} \mathrm{C}$ until total weight loss.

\section{Resistant starch}

RS was measured by the official method AACC 32-40.01/ AOAC 2002.02. The method was carried out with a Megazyme Kit (Megazyme International Ireland Ltd). In this method, approximately $50 \mathrm{~g}$ of each sample was grinded to pass through a $1.0 \mathrm{~mm}$ sieve. All materials were transferred to a wide-mouthed plastic jar and mixed well by shaking and inversion. This method is based on removal of nonresistant starch. An aliquot of sample material $(100 \mathrm{mg})$ was combined with sodium maleate buffer ( $\mathrm{pH}$ 6.0) containing pancreatic $\alpha$-amylase and amyloglucosidase (AMG). Sample was mixed and incubated in a shaking water bath for $16 \mathrm{~h}$ at $37{ }^{\circ} \mathrm{C}$, during which time nonresistant starch was solubilized and hydrolyzed to D-glucose by the combined action of the two enzymes. The reaction was stopped by adding ethanol $(99 \% \mathrm{v} / \mathrm{v})$. The resulting mixture was then washed twice by suspension in aqueous ethanol ( $50 \% \mathrm{v} / \mathrm{v})$, followed by centrifugation at $3000 \mathrm{rpm}$ for $10 \mathrm{~min}$. Free liquid was removed, and the pellet (RS) was dissolved in $2 \mathrm{M} \mathrm{KOH}$ in an ice/water bath by vigorously stirring for $20 \mathrm{~min}$. The solution was neutralized with sodium acetate buffer, and the starch was hydrolyzed to glucose with AMG 
Table 3 Moisture and resistant starch content in the differently formulated bread products

\begin{tabular}{lll}
\hline Products & Moisture $(\%)$ & RS $(\mathrm{g} / 100 \mathrm{~g} \mathrm{dwb})$ \\
\hline REF & $35.4(0.65)$ & $1.18(0.02)^{\mathrm{ab}}$ \\
LW & $33.0(2.43)$ & $1.08(0.04)^{\mathrm{a}}$ \\
HW & $36.5(2.68)$ & $1.39(0.07)^{\mathrm{c}}$ \\
S25 & $35.8(0.53)$ & $1.24(0.04)^{\mathrm{b}}$ \\
S50 & $35.3(0.93)$ & $1.24(0.17)^{\mathrm{b}}$ \\
LA & $31.1(0.29)$ & $1.23(0.05)^{\mathrm{b}}$ \\
SD & $35.1(2.52)$ & $1.05(0.10)^{\mathrm{a}}$ \\
\hline
\end{tabular}

Data expressed as mean of four samples, values in parentheses are standard deviations

$d w b$ dry weight basis, $R E F$ traditional recipe, $L W$ 2.0:1 flour-to-water ratio, $H W$ 1.4:1 flour-to-water ratio, $S 2525 \%$ of spelt flour, $S 5050 \%$ of spelt flour, $L A$ addition of lactic acid, $S D$ addition of sourdough

Values in column not sharing the same letters as superscripts are significantly different $(P \leq 0.05)$

Table 4 Moisture and resistant starch content in the different loaf sizes and baking conditions

\begin{tabular}{lll}
\hline Products & Moisture $(\%)$ & RS $(\mathrm{g} / 100 \mathrm{~g} \mathrm{dwb})$ \\
\hline REF & $35.4(0.65)$ & $1.18(0.02)^{\mathrm{a}}$ \\
W35 & $32.6(0.60)$ & $1.21(0.04)^{\mathrm{a}}$ \\
W140 & $37.6(0.58)$ & $1.28(0.05)^{\mathrm{ab}}$ \\
W400 & $40.1(0.06)$ & $1.34(0.04)^{\mathrm{b}}$ \\
T150 & $18.3(0.66)$ & $1.36(0.09)^{\mathrm{bc}}$ \\
T120 & $22.2(1.71)$ & $1.46(0.12)^{\mathrm{c}}$ \\
\hline
\end{tabular}

Data expressed as mean of four samples, values in parentheses are standard deviations

$d w b$ dry weight basis

Values in column not sharing the same letters as superscripts are significantly different $(P \leq 0.05)$

(water bath at $50{ }^{\circ} \mathrm{C}$ for $30 \mathrm{~min}$ ). The samples were centrifuged, and an aliquot was incubated with glucose oxidase/ peroxidase reagent (GOPOD) and incubated at $50{ }^{\circ} \mathrm{C}$ for $20 \mathrm{~min}$. Finally, the absorbance of each solution against the blank was measured at $510 \mathrm{~nm}$. This is a measure of the RS content of the sample.

\section{Statistical analysis}

Statistical analysis was performed using Statistical Package for the Social Sciences (SPSS) version 23.0. Analytical data were expressed as the mean with standard deviation (SD). To study the effects of formulations, loaf size and baking conditions, data were analyzed using analysis of variance (one-way ANOVA). To assess the impact of the storage conditions, data were analyzed using analysis of variance (two-way ANOVA). Least significant differences were calculated by the Duncan's test. Statistical significance was determined at $P \leq 0.05$.

\section{Results}

\section{Influence of the formulation}

Statistical data analysis shows that tested formulations affected significantly the RS content $[F(6,21)=6.955$, $P \leq 0.05]$. The comparison of averages by Duncan test is shown in Table 3.

Resistant starch content in the HW formulation (high amount of water-flour-to-water ratio at 1.4:1) was significantly higher than in the other experimental formulations tested. In this formulation, the RS content was increased by $18 \%$ in comparison with the reference bread; other formulations led to more modest increases of $5 \%$ (S25, S50 or LA) or even decreases close to $8-10 \%$ (LW and SD) in comparison with the RS content in the reference bread.

\section{Influence of the loaf size and baking conditions}

Three different loaf sizes and two different baking temperature/time conditions were tested. Analysis of variance of the data generated shows a significant effect on RS content $[F(5,18)=8.326, P \leq 0.05]$ particularly in the case of the binomial baking temperature/time. Statistically significant differences were also shown between levels within each parameter tested (Table 4).

To our knowledge, this is the first study that investigates the influence of the loaf size on RS content. A greater sample size yields a higher RS content. Bread loaves with about $400 \mathrm{~g}$ exhibited significantly higher levels of RS when compared to the reference bread loaf weight ( $70 \mathrm{~g})$; an increase of $14 \%$ was observed.

Bread baking at $120{ }^{\circ} \mathrm{C} / 4 \mathrm{~h}$ led to bread loaves containing significantly higher amounts of RS than those baked at $150{ }^{\circ} \mathrm{C} / 3 \mathrm{~h}$ which in turn also contain more RS than the bread baked at reference conditions $\left(200{ }^{\circ} \mathrm{C} / 30 \mathrm{~min}\right)$; increases of 24 and $15 \%$ were observed, respectively.

\section{Influence of the storage conditions}

Storage conditions (room temperature, refrigeration temperature and sub-zero temperature) up to 1 week of storage were investigated. Analysis of variance demonstrated that RS content was affected by both storage time $[F(3,39)=6.939, P \leq 0.05]$ and storage temperature $[F(2,39)=6.126, P \leq 0.05]$. There was, however, no significant effect from the storage time $\times$ temperature interaction $[F(6,39)=1.167, P=0.343]$. The differences between treatments are shown in Table 5. 
Table 5 RS content ( $\mathrm{g} / 100 \mathrm{~g}$ dwb) under different storage conditions

\begin{tabular}{lllll}
\hline & $\begin{array}{l}\text { Without } \\
\text { storage }\end{array}$ & $\begin{array}{l}\text { Ambient } \\
\text { temperature }\end{array}$ & $4{ }^{\circ} \mathrm{C}$ & $-20{ }^{\circ} \mathrm{C}$ \\
\hline 0 day & $1.18(0.02)^{\mathrm{ab}}$ & - & - & - \\
1 day - & $1.34(0.13)^{\mathrm{bcd}}$ & $1.34(0.05)^{\mathrm{bcd}}$ & $1.24(0.06)^{\mathrm{abc}}$ \\
3 days - & $1.49(0.06)^{\mathrm{d}}$ & $1.33(0.18)^{\mathrm{bc}}$ & $1.30(0.16)^{\mathrm{bc}}$ \\
5 days - & $1.38(0.07)^{\mathrm{cd}}$ & $1.33(0.05)^{\mathrm{bc}}$ & $1.26(0.06)^{\mathrm{abc}}$ \\
7 days - & $1.26(0.15)^{\mathrm{abc}}$ & $1.12(0.02)^{\mathrm{a}}$ & $1.22(0.13)^{\mathrm{ab}}$ \\
\hline
\end{tabular}

Data expressed as mean of four samples, values in parentheses are standard deviations

Values not sharing the same letters as superscripts are significantly different $(P \leq 0.05)$

In general, the storage at any of the temperatures contributed to increasing the RS content in bread. RS content increased steadily up to 3 days of storage upon which it decreased to levels equivalent to REF bread by 7 days. Resistant starch content in breads stored at ambient temperature for 3 and 5 days was significantly higher than in the REF. The highest RS content (26\% increase) was observed in bread loaves stored at room temperature for 3 days. Storage at freezing temperatures was the condition contributing the least to increased RS formation. Storage at refrigeration temperatures also contributed to increasing the RS content but to a less extent than at room-temperature storage.

\section{Discussion}

Several strategies have been hypothesized for increasing RS in bread. In this study, three approaches were duly studied for this purpose, namely via formulation adaptation, via baking conditions modifications or via different storage conditions assessment.

In what concerns formulation adaptation, the standard recipe (REF) has a flour-to-water ratio at 1.6:1. A change in this ratio to $2: 1$ ( $\mathrm{LW}$ ) tended to decrease the amount of RS, while significantly higher levels of RS were found when the flour-to-water ratio changes to 1.4:1 (HW). Similar observations have been recorded by Siljestrom and Asp [15] where authors observed that a change in flour-to-water ratio from 1.8:1 to $1.4: 1$ increases $\mathrm{RS}$ formation from 0.9 to $1.1 \%$ (dwb). Johansson et al. [19] also detected higher levels of RS in the crumb (higher moisture) than in the crust (lower moisture), which agrees with the findings of this study.

Spelt (Triticum aestivum spp spelta) is a primitive wheat, currently used in healthy foods. Spelt flour contains higher amount of RS, about eight-to tenfold more as compared to common wheat (3.7 vs. $0.4 \%$ ) [25]; however, its replacement for part of the common wheat flour (S25 and S50) did not enable significantly higher levels of RS. Furthermore, an increase in the amount of spelt flour (S25 to S50) did not yield a significant increase in RS. Similar observations have been recorded by other researchers [25, 26]. The RS contents in spelt doughs were still considerably higher than those in common wheat doughs. After baking, RS levels were quite similar in spelt and common wheat bread products. In the case of common wheat bread, during the baking process, RS increased from 0.4 to $1.2 \%$, whereas it decreased from 3.3 to $1.2 \%$ in spelt breads [25]. Similar observations have been recorded by Angioloni and Collar [26] where authors observed analogous RS contents in spelt bread and wheat bread (10 and $9 \mathrm{~g} \mathrm{~kg}^{-1}$, respectively). The high content of RS in spelt flour is, probably, due to RS type 2. During the bread baking, this starch undergoes gelatinization, gets available and does not produce breads with higher RS content. Other studies have found that when replacing part of the wheat flour by modified pea starch, banana, oat, rye, teff or buckwheat flours, the results are different. The RS content of bread was, in general, significantly higher, but bread loaves presented lower overall acceptability [26-28].

Since acidification lowers the digestibility of starch, lactic acid was added directly or indirectly via sourdough preparation. However, the presence of lactic acid, added or produced during sourdough, had no influence on RS formation. Liljeberg et al. [21] reported similar results in barley bread (80\% whole barley flour and $20 \%$ white wheat flour) with the addition of similar concentration of lactic acid and the same baking conditions $\left(200{ }^{\circ} \mathrm{C} / 30 \mathrm{~min}\right)$. All bread products had a similar content of RS (1.3-2.1 g/100 g starch basis). Other researchers have found high RS content in breads added with lactic acid and baked for $20 \mathrm{~h}$ at $120{ }^{\circ} \mathrm{C}$. Liljeberg et al. [16] observed an RS increased from 3.0 to $6.6 \%$ (dwb) in rye bread $(70 \%$ wholemeal rye flour and $30 \%$ white wheat flour) added with $1.6 \mathrm{~g}$ lactic acid (90 wt\%)/100 g flour compared with the same formulation without lactic acid and baked at $40 \mathrm{~min}$ at $200{ }^{\circ} \mathrm{C}$. Similar observations have been recorded by Hallstrom et al. [20] where authors observed that an addition of $1.3 \mathrm{~g}$ lactic acid (90 wt\%)/100 g flour in bread based on a novel wheat genotype with elevated amylose content had no effects at the RS content ( $7 \%$ starch basis) when baked at conventional conditions. The same essay by changing the bread baking conditions $\left(20 \mathrm{~h}\right.$ at $120{ }^{\circ} \mathrm{C}$ ) shows an increase in the RS content from 13 to $19 \%$ (starch basis). The presence of lactic acid might further promote retrogradation of starch during the long-time/low-temperature baking process. Under these conditions, linearization of amylopectin occurs due to the prolonged activity of amylases in the dough, resulting in more linear glucose chains that can retrograde more easily and thereby contribute to RS formation. The linearization might be more prominent in the presence of lactic acid 
[16]. A more recent study refers that the presence of lactic acid could further promote interactions between starch and gluten and, thereby, the formation of slowly digestible and enzyme-resistant starch, also at long-time/low-temperature baking [20]. Under the conditions studied herein, the presence of lactic acid did not affect the formation of RS.

Proportionally, high RS content in larger bread loaves is probably related to the water content of bread and baking temperature. Inside larger loaves, the moisture content is higher and the crumb does not achieve temperatures as high as in the smaller loaf units. There are no known studies that have tested the influence of different loaves sizes on the RS formation, but these results agree with those obtained when changing the flour-to-water ratio $[15,19]$.

The low-temperature/long baking time condition influenced significantly RS formation. These results agree with those obtained by Liljeberg et al. [16] in rye breads (3 vs. $5.4 \%$ starch basis), Akerberg et al. [17] in breads from barley genotypes varying in amylase/amylopectin ratio (0.6-3.5 vs. $2.5-10.3 \%$ starch basis), Yadav [18] in white wheat breads (2.6 vs. $4.2 \% \mathrm{dwb}$ ) and Hallstrom et al. [20] in whole wheat with elevated amylose content breads ( 7 vs. $13 \%$ starch basis). These authors observed that breads baked at $120{ }^{\circ} \mathrm{C}$ for $20 \mathrm{~h}$ showed significantly higher RS content in comparison with breads baked under conditions of higher temperature/lesser baking time. These conditions $\left(120{ }^{\circ} \mathrm{C} / 20 \mathrm{~h}\right)$ are similar to that used to prepare pumpernickel bread, a whole grain rye bread which has been popular for centuries in Northern Europe. Jenkins et al. [29] observed a much higher RS content in the ileal effluent following ingestion of pumpernickel bread. The high RS levels reported are probably due to both increase in amylose retrogradation and amylopectin linearization during the long-time/low-temperature baking process [16]. According to Eerlingen et al. [30], the storage of an autoclaved wheat starch gel at $100^{\circ} \mathrm{C}$ had a high RS yield compared with storage at 0 or $68^{\circ} \mathrm{C}$. There was an induction time of about $150 \mathrm{~min}$ before the RS yields quickly increased to a maximum. During the longtime/low-temperature baking $\left(20 \mathrm{~h} / 120{ }^{\circ} \mathrm{C}\right)$, the temperature in the crumb reaches $100{ }^{\circ} \mathrm{C}$ about $1 \mathrm{~h}$ after loaves are put in the oven [17]. However, the higher amount of RS may also be due to the linearization and subsequent retrogradation of amylopectin as described above, even without the presence of lactic acid [16]. In our study, the exposure to $150{ }^{\circ} \mathrm{C} / 3 \mathrm{~h}$ seems to favor significantly the amount of RS and baking at $120{ }^{\circ} \mathrm{C} / 4 \mathrm{~h}$ yields even higher content of RS (increase of $24 \%$ ). It would be interesting to be able to increase even more the time for bread baking at $120^{\circ} \mathrm{C}$ in order to further increase the RS content.

A significant increase in the RS content was observed when the bread loaves were stored at room temperature for
3 and 5 days. These results at room temperature agree with previous studies by Yadav [18] who showed that RS content increased significantly by $25 \%$ over the control after a storage period of $96 \mathrm{~h}$. A significant increase in the RS content of bread was also observed by Eerlingen et al. [24] after 7 days of storage at the same conditions. The observed increase in RS content during storage for 3 and 5 days was probably due to amylopectin retrogradation. Despite these conditions, it is still necessary to take into account the acceptability by the consumer.

Bread staling is a complex phenomenon involving multiple factors and not completely explained. The most important causes responsible for this alteration are starch transformation, starch-gluten interactions and moisture redistribution. Retrogradation of starch remains the most common factor contributing to bread staling, mainly the amylopectin retrogradation but amylose is also involved. Moisture content and moisture migration are also believed to be a significant factor contributing to bread staling. The moisture transfer from crumb to the crust plays a significant role in firming insofar as promotes the amylopectin retrogradation. Most evidence suggests a loss of water from gluten network via transfer to starch, which enables crystallization of amylopectin once amylopectin retrogradation involves incorporation of water molecules into the crystallites [31, 32]. Other studies in cornbread reported that storage increased the RS content but only up to 4 days, particularly at $4{ }^{\circ} \mathrm{C}$. After 7 days of storage, there was a decrease in RS levels, with lowest levels at $-20{ }^{\circ} \mathrm{C}$. This fact may be a result of reverse in retrogradation of amylopectin [13]. These results are in good agreement with those reported herein where bread RS content was lowest at 7 days of storage at $4{ }^{\circ} \mathrm{C}$.

Earlier studies by Johansson et al. [19], Siljestrom and Asp [15] and Berry [23] have reported somewhat different results. Their studies revealed that in wheat bread, RS was present immediately after baking, yet no increase occurred during storage.

Further research is needed to test all technological variations in the same bread in order to obtain a product with high amount of RS. In what concerns, the water content of the dough and the storage conditions are an evident need for further research to take into account the mechanisms of bread staling. It would also be interesting to investigate whether the bread optimized in RS has high acceptability by consumers and test the health effects, especially reduction in postprandial glycemia response.

\section{Conclusions}

The results of the present study indicate that it is possible to increase wheat bread RS content by modifying the 
ingredients and processing conditions. A higher level of moisture in the dough (flour-to-water ratio at 1.4:1) enhanced the RS content by $18 \%$ over the standard formula. A larger loaf size also resulted in an increase in the RS level. Bread loaf with $400 \mathrm{~g}$ had $14 \%$ more of RS than a bread loaf with $70 \mathrm{~g}$. An extended baking process also favored significantly the formation of RS in bread. In this study, baking the bread at $120{ }^{\circ} \mathrm{C}$ for $4 \mathrm{~h}$ enhanced RS content by $24 \%$. The bread storage conditions also influenced the content of RS: Keeping the bread at room temperature for 3 days seems to be the way to further increase the RS content (over $26 \%$ ).

Acknowledgments The authors would like to thank Pedro Oliveira e Silva, Ph.D., for their assistance in data analysis. All funding for this work was provided by Polytechnic Institute of Beja, Portugal.

\section{Compliance with ethical standards}

Conflict of interest The authors declare that they have no conflict of interest.

Compliance with ethics requirements This article does not contain any studies with human or animal subjects.

\section{References}

1. Belitz H-D, Grosh W, Schieberle P (2009) Food chemistry, 4th revised and extended. Springer, Berlim

2. Englyst HN, Cummings JH (1985) Digestion of the polysaccharides of some cereal foods in the human small-intestine. Am J Clin Nutr 42(5):778-787

3. Asp NG, Bjorck I, Holm J, Nyman M, Siljestrom M (1987) Enzyme resistant starch fractions and dietary fiber. Scand J Gastroentero 22:29-32

4. Englyst HN, Kingman SM, Hudson GJ, Cummings JH (1996) Measurement of resistant starch in vitro and in vivo. Br J Nutr 75(5):749-755

5. Higgins JA, Brown IL (2013) Resistant starch: a promising dietary agent for the prevention/treatment of inflammatory bowel disease and bowel cancer. Curr Opin Gastroen 29(2):190-194

6. Haralampu SG (2000) Resistant starch-a review of the physical properties and biological impact of RS3. Carbohyd Polym 41(3):285-292

7. Sajilata MG, Singhal RS, Kulkarni PR (2006) Resistant starcha review. Compr Rev Food Sci F 5(1):1-17

8. Homayouni A, Amini A, Keshtiban AK, Mortazavian AM, Esazadeh K, Pourmoradian S (2014) Resistant starch in food industry: a changing outlook for consumer and producer. Starch Starke 66(1-2):102-114

9. Fuentes-Zaragoza E, Riquelme-Navarrete MJ, Sanchez-Zapata E, Pérez-Alvarez JA (2010) Resistant starch as functional ingredient: a review. Food Res Int 43(4):931-942

10. Nugent AP (2005) Health properties of resistant starch. Nutr Bull 30(1):27-54

11. Fuentes-Zaragoza E, Sanchez-Zapata E, Sendra E, Sayas E, Navarro C, Fernandez-Lopez J, Pérez-Alvarez JA (2011) Resistant starch as prebiotic: a review. Starch Starke 63(7):406-415

12. Higgins JA (2014) Resistant starch and energy balance: impact on weight loss and maintenance. Crit RevFood Sci 54(9):1158-1166
13. Niba LL (2003) Effect of storage period and temperature on resistant starch and beta-glucan content in cornbread. Food Chem 83(4):493-498

14. Niba LL (2003) Processing effects on susceptibility of starch to digestion in some dietary starch sources. Int J Food Sci Nutr 54(1):97-109

15. Siljestrom M, Asp NG (1985) Resistant starch formation during baking - effect of baking time and temperature and variations in the recipe. Z Lebensm Unters For 181(1):4-8

16. Liljeberg H, Akerberg A, Bjorck I (1996) Resistant starch formation in bread as influenced by choice of ingredients or baking conditions. Food Chem 56(4):389-394

17. Akerberg A, Liljeberg H, Bjorck I (1998) Effects of amylose/ amylopectin ratio and baking conditions on resistant starch formation and glycaemic indices. J Cereal Sci 28(1):71-80

18. Yadav BS (2011) Effect of frying, baking and storage conditions on resistant starch content of foods. Br Food J 113(6-7):710-719

19. Johansson CG, Siljestrom M, Asp NG (1984) Dietary fiber in bread and corresponding flours-formation of resistant starch during baking. Z Lebensm Unters Forsch 179(1):24-28

20. Hallstrom E, Sestili F, Lafiandra D, Bjorck I, Ostman E (2011) A novel wheat variety with elevated content of amylose increases resistant starch formation and may beneficially influence glycaemia in healthy subjects. Food Nutr Res 55:7074

21. Liljeberg HGM, Lonner CH, Bjorck IME (1995) Sourdough fermentation or addition of organic-acids or corresponding salts to bread improves nutritional properties of starch in healthy humans. J Nutr 125(6):1503-1511

22. Siljestrom M, Bjorck I, Eliasson AC, Lonner C, Nyman M, Asp NG (1988) Effects on polysaccharides during baking and storage of bread-in vitro and in vivo studies. Cereal Chem 65(1):1-8

23. Berry CS (1986) Resistant starch-formation and measurement of starch that survives exhaustive digestion with amylolytic enzymes during the determination of dietary fiber. J Cereal Sci 4(4):301-314

24. Eerlingen RC, Vanhaesendonck IP, Depaepe G, Delcour JA (1994) Enzyme-resistant starch 3. The quality of straight-dough bread containing varying levels of enzyme-resistant starch. Cereal Chem 71(2):165-170

25. Abdel-Aal E-SM, Rabalski M (2008) Effect of baking on nutritional properties of starch in organic spelt whole grain products. Food Chem 111(1):150-156

26. Angioloni A, Collar C (2011) Nutritional and functional added value of oat, Kamut ${ }^{(\mathrm{R})}$, spelt, rye and buckwheat versus common wheat in breadmaking. J Sci Food Agr 91(7):1283-1292

27. Juarez-Garcia E, Agama-Acevedo E, Sayago-Ayerdi SG, Rodriguez-Ambriz SL, Bello-Perez LA (2006) Composition, digestibility and application in breadmaking of banana flour. Plant Food Hum Nutr 61(3):131-137

28. Mario Sanz-Penella J, Wronkowska M, Soral-Smietana M, Collar C, Haros M (2010) Impact of the addition of resistant starch from modified pea starch on dough and bread performance. Eur Food Res Technol 231(4):499-508

29. Jenkins D, Jenkins A, Wolever T, Collier G, Rao A, Thompson L (1987) Starchy foods and fiber: reduced rate of digestion and improved carbohydrate metabolism. Scand J Gastroentero 22:32-41

30. Eerlingen RC, Crombez M, Delcour JA (1993) Enzyme-resistant starch.1. Quantitative and qualitative influence of incubationtime and temperature of autoclaved starch on resistant starch formation. Cereal Chem 70(3):339-344

31. Gray A, Bemiller JN (2003) Bread staling: molecular basis and control. Compr Rev Food Sci F 2(1):1-21

32. Fadda C, Sanguinetti AM, Del Caro A, Collar C, Piga A (2014) Bread staling: updating the view. Compr Rev Food Sci F 13(4):473-492 